\title{
Lorentz violation bounds from torsion trace fermion sector and galaxy M 51 data and chiral dynamos
}

\author{
L. C. Garcia de Andrade ${ }^{\mathrm{a}}$ \\ Departamento de Física Teórica, IF-UERJ, Rua São Francisco Xavier 524, Maracanã, Rio de Janeiro, RJ CEP: 20550, Brazil
}

Received: 11 April 2017 / Accepted: 29 May 2017 / Published online: 16 June 2017

(C) The Author(s) 2017. This article is an open access publication

\begin{abstract}
Earlier we have computed a Lorentz violation (LV) bound for torsion terms via galactic dynamos and found bounds similar to the one obtained by Kostelecky et al. (Phys Rev Lett 100:111102, 2008) which is of the order of $10^{-31}$ $\mathrm{GeV}$. Their result was found making use of the axial torsion vector in terms of Dirac spinors and minimal torsion coupling in flat space-time of fermions. In this paper, a torsion dynamo equation obtained using the variation of the torsion trace and galaxy M51 data of 500 pc are used to place an upper bound of $10^{-26} \mathrm{GeV}$ in $\mathrm{LV}$, which agrees with the one by Kostelecky and his group using an astrophysical framework background. Their lowest bound was obtained in earth laboratory using dual masers. One of the purposes of this paper is to apply the Faraday self-induction magnetic equation, recently extended to torsioned space-time, by the author to show that it lends support to physics in Riemann-Cartan space-time, in several distinct physical backgrounds. Backreaction magnetic effects are used to obtain the LV bounds. Previously Bamba et al. (JCAP 10:058, 2012) have used the torsion trace in their teleparallel investigation of the IGMF, with the argument that the torsion trace leads to less weaker effects than the other irreducible components of the torsion tensor. LV is computed in terms of a chiral-torsion-like current in the new dynamo equation analogous to the Dvornikov and Semikoz dynamo equation with chiral magnetic currents. Making use of the chiral-torsion dynamo equation we estimate the LV bounds in the early universe to be of the order of $10^{-24} \mathrm{GeV}$, which was the order of the charged-lepton sector. Our main result is that it is possible to obtain more stringent bounds than the ones found in the fermion sector of astrophysics in the new revised 2017 data table for CPT and Lorentz violation by Kostelecky and Mewes. They found in several astrophysical backgrounds, orders of magnitude such as $10^{-24}$ and $10^{-23} \mathrm{GeV}$ which are not so stringent as the one found here, of $10^{-26} \mathrm{GeV}$, in the torsion fermionic sector with the help of galaxy M51 data. It is also shown that non-
\end{abstract}

a e-mail: garcia@dft.if.uerj.br gauge invariance of chiral dynamos and axial anomalies are obtained from the noninvariance of the electric fields under torsion spatial translations.

\section{Introduction}

Making use of Einstein teleparallelism Bamba et al. [1] have computed an intercluster large-scale magnetic field at $1 \mathrm{Mpc}$ of the order of $10^{-9} \mathrm{G}$ by adiabatic compression, where no galactic dynamo mechanism is needed. They argue that the presence of torsion trace vector components give more appreciable results than using other irreducible components of the torsion tensor in their version of telepallelism. This telepallel theory was first developed by Einstein [2] following Cartan's ideas [3] on a non-Riemannian connection with torsion. The idea stems from a more primitive one, of building an unified theory of gravity and electromagnetism [4]. Several estimates of the torsion have been obtained in laboratory, such as $10^{-17} \mathrm{~cm}^{-1}$ obtained by Laemmerzahl [5] using the Hughes-Drever experiment and its extension of Dirac equation to torsioned space-times. More recently Kostelecky et al. [6] have used dual maser techniques to place bounds on earth lab axial torsion for fermions in flat space-time with torsion, obtaining a similar bound of $10^{-31} \mathrm{GeV}$ for LV. These bounds were obtained on a whole series of physical frameworks collected in a recently revised, big table [7] with LV bounds collected so far in several sectors, such as neutron, quarks, and fermions in general as for example neutrino sectors tables. In this paper we go a step further by making use of a selfinduction magnetic equation in flat space-times with torsion, in order to place bounds on LV using the data of galaxy M51 [8] at 500 pc scales and using its rotation and galactic magnetic fields. Backreaction effects are also addressed. However, it is shown that dynamo amplification is not needed and the amplification of galactic magnetic fields can be made by filaments of plasma under the effect of turbulence [9]. More 
recently Dvornikov and Semikoz [10] have shown that chiral magnetic fields in the early universe may need a turbulent dynamo. This idea is applied here to chiral currents generated by the minimal coupling of torsion to flat space-time and by Ampère and Faraday laws of the Maxwell equations and Ohm's law to yield a new dynamo equation with torsion, due to chiral-torsion-like currents. Again this equation is used to determine the LV bounds in the universe. This paper is organized as follows: in Sect. 2 we review the torsion tensor framework and its irreducible components [11-13]. In Sect. 3 we compute, from galactic data and the self-induction equation, the upper LV bound of $|T| \leq 10^{-26} \mathrm{GeV}$. Section 4 addresses the chiral-torsion-like currents induced from minimal torsion coupling. In Sect. 5 we show that some quantities associated to axial anomalies and chiral dynamos are modified by torsion since as shown in this section the electric fields are not invariant under torsion translations. Discussions and conclusions are left to Sect. 5 .

\section{Riemann-Cartan geometry and Lorentz symmetry breaking}

In this section we revisit the splitting of the torsion tensor $T_{\alpha \beta \gamma}$ into its axial vector part $A^{\alpha}=\frac{1}{6} \epsilon^{\alpha \beta \gamma \delta} T_{\beta \gamma \delta}$, where $\alpha=0,1,2,3$ and the torsion trace vector

$\frac{1}{3}\left[g_{\alpha \beta} T_{\gamma}-g_{\alpha \gamma} T_{\beta}\right]$.

Here the total torsion tensor can be split in irreducible parts:

$T_{\alpha \beta \gamma}=\frac{1}{3}\left[g_{\alpha \beta} T_{\gamma}-g_{\alpha \gamma} T_{\beta}\right]-\frac{1}{6} \epsilon^{\alpha \beta \gamma \delta} T_{\beta \gamma \delta}+M_{\alpha \beta \gamma}$

where $M$ is a mixed component. According to Kostelecky et al. [6], the LV bounds are the same, either when the axial part or the trace-free part are used. Therefore, here we shall choose to use the trace-free vector part since according to Bamba et al. [1] it is the best part of torsion to be used to deal with large-scale magnetic fields. Another important issue discussed in the next section, contrary to previous work, is that it is not necessary to use galactic dynamos to investigate astrophysical bounds in galaxies. Before we end this section a couple of issues need to be addressed: What is the kind of LV violation used here? In this paper we are not using the spontaneous symmetry breaking of a non-conformal breaking photon, which yields a galactic dynamo seed in the form of a primordial magnetic field (PMF) as given by Bertolami and Mota [14]. Here we consider explicitly LV as used by Kostelecky. The torsion constant vector given in the background space-time plays a fundamental role, though models in Riemann-Cartan space-time can be associated with the SSB in LV. The second feature is that here we are using a backreaction feature of the magnetic field in LV model, not necessarily in the inflationary era, where it naturally appears. We refer the reader for a detailed discussion of this problem to Campanelli [15]. In his paper the Ratra model is discussed using the inflaton and LV in such a way that the backreaction problems could be avoided.

\section{Torsion trace gravity sector of LV from M51 galaxy data}

In this section we show that it is not necessary to have a galactic dynamo mechanism to obtain a torsion LV bound as in Ref. [10]. Actually it is shown that, when the galaxy M51 data at $500 \mathrm{pc}$ scales and the magnetic self-induction equation extended to space-times with space-time trace part of torsion are used, one obtains [11]

$\partial \mathbf{B}=\nabla \times(\mathbf{v} \times \mathbf{B})+\eta[\Delta \mathbf{B}-(\nabla . \mathbf{T}) \mathbf{B}]$.

The axial torsion vector couples to the fermion spinor as described by the expression

$A^{\mu} \sim \bar{\psi} \gamma_{5} \gamma^{\mu} \psi$

where $\mathrm{T}$ represents the Cartan torsion trace part vector and $\eta \sim 10^{26} \mathrm{~cm}^{2} \mathrm{~s}^{-1}$ is the Ohmic resistivity. In the M51 galaxy the magnetic fields are not amplified by the dynamo mechanism but actually by the turbulent plasma action on magnetic filaments and its associated contraction [9]. Let us consider the expression of the speed $\mathrm{v}$ in terms of the vorticity $\omega$ of the galaxy rotation, considered as $\sim 10^{-6} \mathrm{~Hz}$. Thus the complete self-induction magnetic field equation reads

$\partial_{t} \mathbf{B}=\eta[\Delta \mathbf{B}-(\nabla . \mathbf{T}) \mathbf{B}]+\nabla \times[[\omega \times \mathbf{d}] \times \mathbf{B}]$.

Let us now expand the magnetic induction vector field $\mathbf{B}$ in terms of the Taylor expression,

$\mathbf{B}(\mathbf{x}, t) \approx \mathbf{B}\left(\mathbf{X}, t_{0}\right)+\left.\partial_{t} \mathbf{B}\right|_{t_{0}}(\Delta t)$.

Substitution of the so-called dynamo equation into the mean value theorem of calculus of partial derivatives in Eq. (6) yields

$$
\begin{aligned}
& \mathbf{B}(\mathbf{x}, t) \approx \mathbf{B}\left(\mathbf{X}, t_{0}\right)+\eta[\Delta \mathbf{B}-(\nabla . \mathbf{T}) \mathbf{B}] \\
& +[\nabla \times[\omega \times \mathbf{d}] \times \mathbf{B}] \Delta t .
\end{aligned}
$$

Considered dimensionally, the last equation reads

$\omega=\frac{\eta}{d^{2}}\left[1-T^{\max }{ }_{M 51} d_{G}\right]$,

which is a dispersion relation. One important aspect in this derivation is that we use the backreaction magnetic contrast $\left|\frac{\delta B}{B}\right| \sim 1$ which from the above data of M51 galaxy yields 


$$
T^{M 51} \leq 10^{-26} \mathrm{GeV} \text {. }
$$

Since Kostelecky et al. set the limit [6] for astrophysical bounds of $\mathrm{LV}$ at $|A| \sim 10^{-27} \mathrm{GeV}$, one may say that our result is confirmative. Not only their estimate is correct, but it also shows how useful astronomical data and dynamolike equations in modified gravity sectors are in placing new bounds on LV. Besides, our result in Eq. (9) is more stringent by ten orders of magnitude, than the Kostelecky one. For example, recently, the Finsler geometry has been used to place bounds in LV [16]. It is also interesting to compare the other bounds obtained by other authors with respect to the bound obtained for LV in this paper. A very interesting example of more stringent bound for the neutron sector of LV was obtained by Walsworth [17] in the laboratory, using tests of CPT and LV with hydrogen and noble-gas masers, where they obtained the bound $10^{-27} \mathrm{Gev}$ similar to ours here instead of the $10^{-31} \mathrm{GeV}$ bound. Another sort of bound on LV torsion was obtained by Mohanty and U. Sarkar using $\mathrm{K}$-mesons to obtain averaged components of the $\mathrm{LV}$ torsion zero-component averaged as $\left\langle T^{0}\right\rangle \leq 10^{-25} \mathrm{GeV}$ [18]. Yet another attempt of the bound LV has been made earlier by the author [19] where use was made of a CP-violation $\alpha^{2}$ dynamo in an axion photon cosmic plasma, obtaining a lower bound of $10^{-32} \mathrm{GeV}$ for the same torsion zero-component. Even for radio galaxies, it is possible to use large-scale magnetic fields to place bounds in LV [20], using the polarization of radio galaxies leading to a $\mathrm{LV}$ bound of $T \sim 1.7 \times 10^{-46}$ $\mathrm{GeV}$, which are more stringent limits than the ones found by Kostelecky and Mewes [21], of the order of $10^{-43} \mathrm{GeV}$, using WMAP data.

\section{Chiral-torsion-like magnetic currents and new dynamo equation and LV bounds}

Very recently, Dvornikov and Semikoz [10] have investigated the magnetohydrodynamics (MHD) of magnetic plasmas taking into account chiral magnetic effects (CME) and obtained the dynamo turbulent effects and magnetic field instability coming from the CME. They also get the modified Faraday self-induction equation with implications to the early universe. By analogy, from the Ampère equation and its magnetic currents obtained from extending the spacetime to torsioned space-time, throughout minimal coupling $\nabla \rightarrow \nabla+\mathbf{T}$ and Ohm's equation, we have

$\mathbf{J}_{\mathrm{Ohm}}=\sigma_{\mathrm{cond}}[\mathbf{E}+(\mathbf{v} \times \mathbf{B})]$.

Here the electric conductivity is $\sigma_{\text {cond }}=\sigma_{\mathrm{e}} T$, where the Kelvin temperature $T$ is not to be confused with the Cartan torsion. From Ampère's law
$\mathbf{J}_{\mathrm{Ohm}}=\nabla \times \mathbf{B}$.

Then substitution of the minimal coupling relation into the Ampère equation yields

$\mathbf{J}_{\mathrm{Ohm}}-\mathbf{T} \times \mathbf{B}=\nabla \times \mathbf{B}$.

Note that the LHS net current contains a term proportional to the magnetic field $\mathbf{B}$ whose coefficient of proportionality is the exact torsion. This is analogous to the chiral current given by [22]

$\mathbf{J}_{\mathrm{CME}-\text { torsion }} \sim-\mathbf{T} \times \mathbf{B}$.

To compare with the $J_{\mathrm{CME}}$ chiral current we reproduce here the chiral current as given in Ref. [10] by

$\mathbf{J}_{\mathrm{CME}}=2 \alpha_{\mathrm{em}} \frac{\mu_{5}}{\pi} \mathbf{B}$.

Of course the chiral current is proportional to the magnetic field and the chiral torsion would only be $\mathbf{T} \times \mathbf{B} \sim \mathbf{B}$. Note from the simple vectorial identity $\mathbf{B} . \mathbf{T} \times \mathbf{B} \sim B^{2}$, that it vanishes, which would imply that the magnetic energy induced by torsion would vanish, therefore to deduce the dynamo chiral-torsion equation below the analogy cannot be a perfect method. Let us now proceed with the derivation of the dynamo equation in this chiral-torsion framework, by firstly using the Faraday equation,

$\partial_{t} \mathbf{B}=-\nabla \times \mathbf{E}$.

Substitution of Ohm's law into this equation and using the appropriate magnetic and electric currents above one obtains the new dynamo equation with torsion, by taking into account the minimal coupling of the Cartan torsion,

$\partial_{t} \mathbf{B}=\nabla \times(\mathbf{v} \times \mathbf{B})+\eta_{m}[\Delta \mathbf{B}-\mathbf{T} . \nabla \times \mathbf{B}]-\mathbf{T} \times \mathbf{v} \times \mathbf{B}$

where the first term on the RHS of this dynamo, the "chiral" torsion equation in flat space-time plus torsion, is the advection dynamo term. In analogy to the previous section, we shall derive the dispersion relation to obtain the bounds on the torsion corresponding to LV in the early universe. After some simple algebra one obtains

$\omega \sim \frac{1}{\lambda} \Omega \lambda+\frac{\eta}{\lambda^{2}}-T \Omega \lambda-\frac{\eta}{\lambda} T$

where we have used the velocity in the hot plasma of the early universe as $v \sim \Omega \lambda$. The term B for the magnetic field was obviously canceled from both sides of the equation, since they are present in each of their terms on both sides of the dynamo equation. Let us now perform the estimate in 
question using the following data for the early universe: The coherent length is

$\lambda \sim 5 \mathrm{pc}$.

The Kelvin temperature is $10^{2} \mathrm{MeV} \leq T \leq 10^{2} \mathrm{GeV}$, and the electron conductivity in the electron plasma is $\sigma_{\mathrm{e}} \sim 100$. First, to compute the torsion T, one solves Eq. (17) by taking $\omega \sim \Omega$, where the vorticity is given by $\Omega \sim 10^{-16} \mathrm{~Hz}$. Within these approximations one obtains from the dispersion equation the expression for the torsion:

$T \sim \eta\left[\lambda^{2}-\Omega \lambda-\frac{\eta}{\lambda}\right]^{-1}$.

Now by making use of this section's data, one obtains the $\mathrm{LV}$ bound for torsion as $|\mathbf{T}| \sim 10^{-24} \mathrm{GeV}$, which in the Kostelecky-Russell table [7] corresponds to the LV bound for the charged-lepton sector, which translated in terms of the early universe would be the electroweak sector.

\section{Chiral dynamos and axial anomalies from torsion in cosmology}

In the last section, we note that magnetic fields are torsion translation invariants to some physical quantities, which shall be seen in this section not to affect the magnetic helicity density $h \sim \mathbf{A} . \mathbf{B}$, which is a physical fundamental quantity for chiral dynamos. However, due to recent work of Rogachevskii et al. [23] on turbulent and chiral dynamos from massless fermions, we show that torsion plays an important role in axial anomalies in cosmology, since the electric field $\mathbf{E}$, given by

$\mathbf{E}=\nabla \phi-\frac{1}{c} \frac{\partial \mathbf{A}}{\partial t}$,

is non-invariant under translations. Actually it is not gauge invariant; in a similar manner Kostecky et al. showed that Lorentz symmetry is violated under torsion. Let us first show that torsion translations $\nabla \rightarrow \nabla-\mathbf{T}$ make the magnetic helicity density invariant:

$h_{\text {torsion }} \sim \mathbf{A} . \mathbf{B}=h_{\text {Eucl }}-\mathbf{A} .[\mathbf{T} \times \mathbf{A}]=h_{\text {Eucl }}$,

since the second term on the RHS of the expression above vanishes. However, there it is possible to show that torsion still may contribute, indirectly, to chiral dynamos, since the electric field is not invariant under the torsion translation such as

$\mathbf{E}_{\text {torsion }}=\mathbf{E}-\mathbf{T} \phi$ where $\phi$ is the electric potential. Thus, in some equations as the one of chiral anomalies we have current conservation,

$\frac{\partial n_{5}}{\partial t}+\nabla \cdot \mathbf{J}_{5}=\frac{2 e^{2}}{\pi h^{2} c} \mathbf{E} . \mathbf{B}$.

Therefore, this equation when associated to torsioned spacetime becomes

$$
\frac{\partial n_{5}}{\partial t}+\nabla . \mathbf{J}_{5}-\mathbf{T} . \mathbf{J}_{5}=\frac{2 e^{2}}{\pi h^{2} c}[\mathbf{E} . \mathbf{B}-\mathbf{T} . \mathbf{B}]
$$

Thus the source term is not gauge invariant as well as in axial anomaly. In the next example we consider the equation of a chiral anomaly given by Rogachevskii et al. [23], where they introduced a new term in the electric current proportional to the chiral chemical potential $\mu_{5}$, conjugated to the axial charge, in the usual thermodynamical sense. This term has the form

$$
\langle\mathbf{J}\rangle=\frac{e^{2}}{\pi h^{2} c}\left(\mu_{L}-\mu_{R}\right) \mathbf{B}=\frac{\alpha_{m}}{\pi h} \mu_{5} \mathbf{B}
$$

where the $\mathrm{L}$ and $\mathrm{R}$ indices mean, respectively, the left and right handed chiralities. Here $\frac{\mu_{L}}{\mu_{R}}$ is the chemical potential. In the inhomogeneous case the expression reads

$$
\langle\mathbf{J}\rangle \rightarrow J_{\Theta}=\mathbf{B} \partial_{t} \Theta+c \nabla \Theta \times \mathbf{E}
$$

where $\Theta$ is the inhomogeneous factor. Note that in this case the chiral magnetic current does possess a real contribution of the Cartan torsion vector like

$J_{\Theta \text { torsion }}=J_{\Theta}-c \nabla \Theta \times \mathbf{T} \phi+c \mathbf{T} \Theta \times \mathbf{E}$.

Thus torsion induces two new extra terms in the chiral anomaly current, in the case of inhomogeneity. Physical implications of these extra current terms due to torsion may be interesting consequences, the study of which shall be left for a future paper. This generalization can be applied to quark-gluon plasmas. Another interesting application of the axial anomaly in space-times with torsion is in the expanding universe where the conservation equation becomes

$\frac{1}{\sqrt{-g}}\left[\partial_{\mu}\left(\sqrt{-g} j^{\mu}\right)\right]=\frac{\alpha_{\mathrm{em}}}{4 \pi h} \epsilon^{\mu \nu \lambda \rho} F_{\mu \nu} F \lambda \rho ;$

$\epsilon$ is a flat-space stress-energy tensor and $\mathrm{g}$ is the determinant of the pseudo-Riemannian metric. Using definitions $\mathbf{E}$ and $\mathbf{B}$ like in Eq. (21) one found

$\epsilon^{\mu \nu \lambda \rho} F_{\mu \nu} F_{\lambda \rho}=8 \pi a(t)^{3} \mathbf{E} . \mathbf{B}$ 
Then the expression for the current becomes

$$
\frac{1}{a^{3}} \partial_{t}\left(a^{3} n_{5}\right)+\nabla . \mathbf{J}_{5}=\frac{2 \alpha_{e m}}{\pi h} a^{3} \mathbf{E} . \mathbf{B} .
$$

Note, for example in the last expression, the axial current is extended to torsioned space-time and it seems that a massive vector-like field appears on the LHS of equation, while in the other side, there is an electric translation due to torsion; on the cosmological level in space-times endowed with torsion, the gauge invariance is broken, similarly to Lorentz symmetry breaking. Explicitly one may observe that the new torsioned space-time extended equation (31) yields

$$
\frac{1}{a^{3}} \partial_{t}\left(a^{3} n_{5}\right)+\nabla \cdot \mathbf{J}_{5}+\mathbf{J}_{5} \cdot \mathbf{T}=\frac{2 \alpha_{e m}}{\pi h} a^{3}[\mathbf{E} \cdot \mathbf{B}+\phi \mathbf{B} . \mathbf{T}] .
$$

Note from these equations that, when the torsion vector is severely constrained according to the expressions

$$
\mathbf{J}_{5} \cdot \mathbf{T}=0
$$

and

$\mathbf{B} . \mathbf{T}=0$,

one obtains again the gauge invariant expression (30). Note, however, that subtracting these two constraint torsion expressions one obtains

$\left[\mathbf{J}_{5}-\mathbf{B}\right] . \mathbf{T}=0$.

Then one notices that the chiral dynamo or chiral magnetic field relation (14) yields

$$
\left(2 \alpha_{\mathrm{em}} \frac{\mu_{5}}{\pi}-1\right)[\mathbf{B} . \mathbf{T}]=0 .
$$

As long as the term inside the round brackets does not vanish, the constraint (33) is fulfilled. An analogous expression with torsion terms may appear in this equation in universes endowed with space-time torsion. Cosmological consequences of this framework may appear elsewhere.

\section{Conclusions}

In this paper we discuss the bounds for LV symmetry based on galactic astrophysics and early universe data. It is interesting to note that in [11] we derived a dynamo flat space-time torsioned extension of GR dynamo equation based on the Lagrangian, or action, for the parity-violating curvature. In the case of the coupling of an axion field to the space-time curvature the term $\frac{1}{2} \epsilon^{\mu \nu \alpha \beta} R_{\mu \nu \sigma \tau} R_{\alpha \beta} \sigma \tau$, where $R_{\alpha \beta \nu \sigma}$ is the Riemann tensor, should be included in the analysis of the early universe, which was neglected in the paper of Boyarsky et al. [15]. These are exactly the part of parity violation we consider to derive the dynamo equation in the torsion case. The only difference is that our curvatures were of Riemann-Cartan space-time type. It would be interesting to take into account other model dependent chiral magnetic field dynamo equations by considering instead the Riemann-Cartan curvatureviolating terms in the LV bounds search. We plan to investigate this issue soon. Several other attempts of placing limits to $\mathrm{LV}$ were discussed in the text with several sources as $K$ mesons, $\mathrm{CP} \alpha^{2}$-dynamos, radiogalaxies magnetic fields and even in laboratory for LV in the neutron sector. It seems our results are compatible with all these results. Our main result in this report is that our LV torsion bound is more stringent than the ones found in the fermion sector so far; therefore they are appropriate as a source for torsion, of astrophysics in the new revised 2017 data table for CPT and Lorentz violation by Kostelecky and Mewes. Several results were found by them in astrophysical settings with astrophysical orders of magnitude for $\mathrm{LV}$ such as $10^{-24}$ and $10^{-23}, \mathrm{GeV}$, which are not so stringent as the one found here in the torsion fermionic sector with the help of galaxy M51 data, which is of the order of $10^{-26} \mathrm{GeV}$. After we finished the first draft of this paper we found an interesting paper by Rogachevskii et al. [23], where they addressed a detailed theory of MHD turbulence and dynamos with chiral magnetism besides the chiral laminar dynamos including the backreaction discussed and used in this paper. In their paper they have investigated the chiral MHD equations, which includes a backreaction of the magnetic field on the chemical potential. They showed that chiral MFs decrease the frequency of Alfvén waves for incompressible flows; in addition to the laminar chiral dynamo effect, there is a dynamo mechanism caused by the action of the joint velocity of the shear and chiral magnetic dynamo. It is exactly at this point that we can make use of the recent relation between dynamo shear and Cartan torsion discussed in [24], where the torsion mimics shear, with a chiral magnetic field. They also investigated the relation between chiral magnetic field and $\alpha$-effect. This is another point of contact between torsion and chiral magnetic effects, since we have investigated recently the relation between CP-dynamos and torsioned space-time [19], used to place bounds on LV symmetry. Analogous implications for the relations between chiral magnetic fields and torsion throughout LV in the early universe may appear elsewhere. Therefore we must conclude that besides the more stringent LV bounds due to torsion, we have shown that torsion translation also breaks the gauge symmetry invariance of some important equations for chiral dynamos of massless fermions. Cosmological implications may appear elsewhere.

Acknowledgements We would like to express our gratitude to V.A. Kostelecky and his group for helpful discussion of the subject of this 
letter and for his warm hospitality at department of physics at Indiana University, Bloomington. Useful correspondence as regards astronomical data and also discussions with Elisabeth Gouveia dal Pino of turbulent dynamos are grateful acknowledged.

Open Access This article is distributed under the terms of the Creative Commons Attribution 4.0 International License (http://creativecomm ons.org/licenses/by/4.0/), which permits unrestricted use, distribution, and reproduction in any medium, provided you give appropriate credit to the original author(s) and the source, provide a link to the Creative Commons license, and indicate if changes were made. Funded by SCOAP ${ }^{3}$.

\section{References}

1. K. Bamba, C.-Q. Geng, L.-W. Luo, JCAP 10, 058 (2012)

2. A. Einstein, Annalen der math. Band 102, 685 (1930)

3. V. de Sabbata, C. Sivaram, Spin and Torsion and Gravitation (World Scientific, Singapore, 1995)

4. E. Cartan, A. Einstein, Letters on Absolute Parallelism, 1929-1932, ed. by R. Debever (Princeton University Press, Princeton, 1979)

5. C. Laemmerzahl, Phys. Lett. A 228, 223 (1997)

6. A. Kostelecky, N. Russell, J. Tasson, Phys. Rev. Lett. 100, 111102 (2008)

7. A. Kostelecky, N. Russell, Data Tables for Lorentz and CPT violation revised 2017 version published in Los Alamos (2017). arXiv:0522.0581

8. U. Klein, A. Fletcher, Galactic and Intergalactic Magnetic Fields (Springer, Berlin, 2015)
9. E.G. Dal Pino, private correspondence (2017)

10. M. Dvornikov, V.B. Semikoz, Influence of the turbulent motion on the chiral magnetic current in the Early universe (2017). arXiv:1612.05897v3 [astro-ph.CO]

11. L.C. Garcia de Andrade, Phys. Lett. B 711, 143 (2012)

12. L.C. Garcia de Andrade, Nucl. Phys. B 847, 485 (2011)

13. L.C. Garcia de Andrade, Phys. Lett. B 468, 28 (2011)

14. O. Bertolami, D. Mota, Phys. Lett. B 455, 96 (1999)

15. L. Campanelli, Eur. Phys. J. C 75, 278 (2015)

16. N. Russell, Phys. Rev. D 91, 045008 (2015)

17. R. Walsworth, in Proceedings of the Third Meeting on CPT and Lorentz symmetry breaking, Bloomington, Indiana, ed. by V.A. Kostelecky (2004)

18. S. Mohanty, U. Sarkar, Constraints on background torsion from K-physics. arXiv:hep-th/9804259v1

19. L.C. Garcia de Andrade, Mod. Phys. Lett. A 26(38), 2863 (2011)

20. L.C. Garcia de Andrade, Phys. Rev. D 84(Brief Reports), 127504 (2011)

21. V.A. Kostelecky, M. Mewes, Astrophys. J. 689, L1 (2008)

22. A. Boyarsky, J. Froelich, O. Ruchayskiy, Magnetohydrodynamics of Chiral Relativistic Fluids (2015). arXiv:1504.04854v1 [hep-ph]

23. I. Rogachevskii, O. Ruchayskiy, A. Boyarsky, J. Froelich, N. Kleeorin, A. Brandenburg, J. Schober, Laminar and Turbulent Dynamos in Chiral MHD-I: Theory (2017). arXiv:1705.00378v1 [physics.plasma-ph]

24. L.C. Garcia de Andrade, Class. Quantum Gravity 33(2), 025006 (2016) 